\title{
Hemogasometria de eqüinos submetidos à obstrução experimental do duodeno, ileo e cólon maior
}

\author{
Blood gas analysis of horses submitted to experimental obstruction of duodenum, \\ ileum and large colon
}

\section{Paula Alessandra Di Filippo ${ }^{\mathrm{I}, *}$ Aureo Evangelista Santana ${ }^{\mathrm{II}}$ Maristela de Cássia Seudo Lopes João Henrique PerottaI Rodrigo Norberto Pereira' ${ }^{\mathrm{I}}$ Gener Tadeu Pereira ${ }^{\mathrm{III}}$}

\section{RESUMO}

Visando estudar os parâmetros do equilíbrio ácidobase de eqüinos submetidos a um modelo experimental de obstrução intestinal, 24 animais foram distribuídos em quatro grupos, controle instrumentado (GI), obstrução do duodeno (GII), íleo (GIII) e cólon maior (GIV). As amostras de sangue venoso foram coletadas antes das cirurgias (T0), durante as obstruções (T30ob-T180ob) e após as desobstruções (T60desT180des). Os eqüinos do GIV, no T30ob, e os eqüinos do GII, nos T60ob, T90ob e T120ob, apresentaram aumento do $\mathrm{pH}(\mathrm{v})$, da $\mathrm{CHCO}_{3}(v P)$ e da cBase (v) que, acrescidos do aumento da $\mathrm{pCO}_{2}(\mathrm{v})$ e da $\mathrm{ctCO}_{2}(\mathrm{v})$, caracterizou a alcalose metabólica com compensação respiratória. Nos T90ob e T120ob, nos animais do GII, e no T180ob, nos animais do GIII, a $\mathrm{pO}_{2}(\mathrm{v})$ e a $\mathrm{SO}_{2}(v)$ tiveram comportamento semelhante. Os valores baixos apresentados pelos animais do GII foram associados à hipercapnia ou à hipoventilação, desencadeadas para a correção da alcalose metabólica. Entretanto, a hipoxemia apresentada pelos animais do GIII foi associada à hipovolemia presente neste período. As alterações ácido-base observadas constituem-se de alterações leves e temporárias, as quais não são capazes de predizer o diagnóstico das obstruções intestinais específicas em eqüinos com cólica. Entretanto, por relacionarem-se diretamente com a precocidade do distúrbio gastrintestinal, elas auxiliam o pesquisador no prognóstico.

Palavras-chave: eqüinos, hemogasometria, obstrução intestinal.

\section{ABSTRACT}

This study aimed to evaluate parameters of acidbase balance in horses submitted to an experimental model of intestinal obstruction. Twenty-four animals were divided in four groups: instrumented control (GI), duodenum obstruction (GII), ileum obstruction (GIII) and large colon obstruction (GIV). Venous blood samples were collected before surgery (T0), during the obstruction (T30ob-T180ob) and after unblocking procedures (T60des-T180des). Animals from GIV, at T30ob and animals from GII, at T60ob, T90ob and T120ob, presented higher values for $\mathrm{pH}(\mathrm{v}), \mathrm{CHCO}_{3}(\mathrm{vP})$ and $c \mathrm{Base}(\mathrm{v})$ which, added to the increase of $\mathrm{pCO}_{2}(\mathrm{v})$ and $\mathrm{ctCO}_{2}(\mathrm{v})$, characterized the metabolic alkalosis with respiratory compensation. At T90ob and T120ob, in GII animals, and at T180ob, in GIII animals the $\mathrm{pO}_{2}(\mathrm{v})$ and $\mathrm{sO}_{2}(\mathrm{v})$ had similar response. Low values presented by GII animals were associated to hypercapnia or hypoventilation triggered for metabolic alkalosis correction. However, a hypoxemia presented by GIII animals was associated to hypovolemia at that period. There were light and temporary acid-base alterations that are not capable to predict specific intestinal obstruction diagnosis in equines with colic. However they help in prognosis since both have a direct relation with the precociousness of gastrointestinal disturbance.

Key words: horse, blood gas analyses, intestinal obstruction.

\section{INTRODUÇÃO}

A homeostase é considerada um dos princípios fundamentais da fisiologia e, entre os muitos processos que ajudam a mantê-la, destaca-se a regulação do equilíbrio ácido-base. Mesmo que as anormalidades ácido-base geralmente não definem um diagnóstico, o restabelecimento do $\mathrm{pH}$ sanguíneo

IPrograma de Pós-graduação em Cirurgia Veterinária, Faculdade de Ciências Agrárias e Veterinárias (FCAV), Universidade Estadual Paulista (Unesp). Endereço para correspondência: Rua professor Julio Bruno, 88, Arnon de Mello, 13560-000, São Carlos, SP, Brasil. E-mail: paula_difilippo@yahoo.com.br. *Autor para correspondência.

IIDepartamento de Clínica e Cirurgia Veterinária, FCAV, Unesp, Jaboticabal, SP, Brasil

IIIDepartamento de Ciências Exatas, FCAV, Unesp, Jaboticabal, SP, Brasil 
normal deve ser considerado no tratamento de qualquer enfermidade (ROBINSON, 2004).

Eqüinos com cólica freqüentemente apresentam-se desidratados e a desidratação é um dos principais fatores responsáveis pelo aparecimento da acidose metabólica nesses animais. A hipovolemia decorrente da desidratação induz à baixa perfusão tecidual, resultando em limitado fornecimento de oxigênio aos tecidos e diminuição na excreção de íon $\mathrm{H}^{+}$pelos túbulos renais. A hipóxia tecidual aumenta a biossíntese do ácido láctico originário do metabolismo anaeróbico (glicólise), liberando-o mais rapidamente do que ele pode ser oxidado ou reconvertido em glicose ou glicogênio pelo fígado, conforme consideraram HJORTKJAER \& SVENDSEN (1979) e GOSSETT et al. (1987).

Estudo realizado por LARSEN (1994) demonstrou que $66,7 \%$ dos eqüinos com cólica apresentaram acidose metabólica. Resultados semelhantes foram obtidos por DI FILIPPO et al. (2008), os quais adicionalmente observaram que os animais que foram a óbito ou foram sacrificados apresentaram acidose metabólica mais intensa. Entretanto, NAPPERT \& JOHNSON, (2001) observaram que a maioria dos eqüinos com cólica não apresentou acidose metabólica acentuada. Além disso, DATT \& USENIK (1975), SVENDSEN et al. (1979) e PUOTUNEN-REINERT \& HUSKAMP (1986) observaram alcalose metabólica, relacionada ao seqüestro de ácido clorídrico no estômago. Por sua vez, DABAREINER \& WHITE (1995) e RIBEIRO FILHO et al. (2007), avaliando eqüinos com compactação de cólon maior, encontraram animais com alcalose e acidose metabólica.

Diante dessas observações, o presente estudo teve o objetivo de avaliar as alterações no equilíbrio ácido-base de eqüinos submetidos à obstrução experimental do duodeno, íleo e cólon maior. O estudo determinou o início e o comportamento em função do tempo de tais alterações e se essas podem ser utilizadas no diagnóstico e no prognóstico de obstruções intestinais especificas em eqüinos com cólica.

\section{MATERIAL E MÉTODOS}

Foram utilizados 24 eqüinos, oito fêmeas (não-gestantes), 16 machos (12 castrados e quatro nãocastrados), sem raça definida, com média de idade de 6,2 $\pm 3,0$ anos, escore corporal de três a quatro (SPEIRS, 1997) e peso corporal médio de $295,9 \pm 32,7 \mathrm{~kg}$. Uma semana antes do experimento, após avaliação clínica com o intuito de avaliar o status sanitário, fez-se o controle de endoparasitas (mebendazol ${ }^{\mathrm{a}}, 50 \mathrm{mg} \mathrm{kg}^{-1}$ ) e de ectoparasitas (deltametrina ${ }^{\mathrm{b}}$ a $\left.0,025 \%\right)$. Os animais foram alojados em piquetes coletivos com dieta à base de feno de coast cross (Cynodon dactylon) e água à vontade. A ração concentrada comercial ${ }^{c}$ foi fornecida duas vezes ao dia em quantidade equivalente a $1 \%$ do peso corpóreo (2,5 a 3,4kg), adicionada de $50 \mathrm{~g} \mathrm{dia}^{-1} \mathrm{de}$ suplemento mineral ${ }^{\mathrm{d}}$.

Os eqüinos foram separados em quatro grupos de seis animais (duas fêmeas, três machos castrados e um não-castrado), sendo que um grupo controle instrumentado ( $\mathrm{G} 1$, sem realização da obstrução intestinal, porém submetidos aos mesmos procedimentos anestésicos e cirúrgicos descritos para os animais dos demais grupos) e três grupos obstruídos. As obstruções intestinais foram realizadas em três diferentes segmentos: duodeno (GII), íleo (GIII) e cólon maior (GIV).

Os animais foram contidos em brete e após tricotomia e antissepsia da fossa paralombar eles foram sedados com acepromazina $1 \%{ }^{\mathrm{e}}\left(0,025 \mathrm{mg} \mathrm{kg}^{-1}, \mathrm{IV}\right)$, cloridrato de xilazina $2 \% \mathrm{f}\left(0,5 \mathrm{mg} \mathrm{kg}^{-1}, \mathrm{IV}\right)$ e meperidina ${ }^{\mathrm{g}}$ (4mg kg-1 IM). Em seguida, foi realizada a anestesia local infiltrativa, utilizando uma associação (1:1) de lidocaína $2 \%^{\mathrm{h}}$ e de bupivacaína $0,75 \%^{\mathrm{i}}$, ambas sem vasoconstritor. Visando mimetizar ao máximo as condições naturais, os animais ensaiados não foram submetidos a jejum hídrico e alimentar prévios.

Com os animais em estação, por meio da laparotomia, flanco direito para duodeno e íleo, esquerdo para cólon maior, os segmentos intestinais foram identificados e, em seguida, um dreno de Penrose no3 foi posicionado ao redor da alça intestinal e após o seu fechamento iniciou-se a obstrução intestinal, segundo modelo descrito por DATT e USENIK (1975). Neste exato momento os animais receberam $1,5 \mathrm{mg} \mathrm{kg}^{-1}$, IV de cloridrato de tramadol ${ }^{\mathrm{j}}$ que, diferentemente dos demais analgésicos opióides, não provoca alterações cardiovasculares ou respiratórias relevantes e não afeta a motilidade nem a atividade metabólica intestinal (NATALINI \& ROBINSON, 2000; BRONDANI et al., 2003). Depois, procedeu-se a sutura simples contínua dos músculos transversos do abdômen e da pele, utilizando-se de vicryl no 2-0 e nylon nํㅜ, respectivamente. As obstruções foram mantidas por três horas e após este período foi promovida a reversão das obstruções, tendo como acesso cirúrgico e protocolo os mesmos utilizados para promovê-las. Os drenos foram então removidos e as cavidades abdominais foram fechadas de acordo com a técnica descrita por TURNER \& McILWRAITH (2002). Durante os períodos de obstrução e de desobstrução, apenas os animais dos grupos GII e GIII apresentaram, como conseqüência da lesão entérica, aumento da freqüência 
cardíaca e respiratória, atonia intestinal e sinais de dor abdominal.

No pós-operatório, os animais receberam flunixim meglumine ${ }^{\mathrm{k}}\left(0,5 \mathrm{mg} \mathrm{kg}^{-1}, \mathrm{IM} / 24 \mathrm{~h} / 2 \mathrm{dias}\right)$, penicilina benzatina ${ }^{1}\left(30.000 \mathrm{UI} \mathrm{kg}^{-1}, \mathrm{IM} / 48 \mathrm{~h} / 3 \mathrm{X}\right)$ e curativos diários da ferida cirúrgica (polivinilpirrolidona-iodo tópica a 1\%). Durante esse período, com exceção de dois animais que apresentaram contaminação da ferida cirúrgica, não foram observadas alterações clínicas relevantes.

Para a análise hemogasométrica, as amostras de sangue foram coletadas anaerobicamente, por meio da punção da jugular, com agulha $25 x 8$, em seringas plásticas descartáveis de $1 \mathrm{~mL}$, previamente heparinizadas ${ }^{\mathrm{m}}$ e acondicionadas em água com gelo e encaminhadas imediatamente (com intervalo de, no máximo, três minutos) para a análise das seguintes variáveis: $\mathrm{pH}$ - $\mathrm{pH}(\mathrm{v})$, pressão parcial de oxigênio $\mathrm{pO}_{2}(\mathrm{v})$, pressão parcial do dióxido de carbono - $\mathrm{pCO}_{2}(\mathrm{v})$, concentração de bicarbonato no plasma - $\mathrm{CHCO}_{3}^{-}(\mathrm{vP})$, concentração de base titulável - cBase(v), concentração total de dióxido de carbono no plasma do sangue venoso - $\mathrm{CtCO}_{2}(\mathrm{v})$ e saturação de oxigênio - $\mathrm{sO}_{2}(\mathrm{v})$. Essas análises foram realizadas em aparelho de hemogasometria ${ }^{\mathrm{n}}$, com calibração automática, segundo os intervalos: T0 (basal), T30ob, T60ob, T90ob, T120ob, T150ob e T180ob minutos (correspondente à fase de obstrução) e T60des, T120des e T180des minutos (correspondente à fase de desobstrução). Nestes mesmos períodos amostras de sangue e de líquido peritoneal foram obtidas para o estudo do perfil hematológico, bioquímico e eletroforético. Entre os animais dos grupos GII e GIII, foi revelada uma resposta inflamatória mais intensa, caracterizada por maiores contagens global e diferencial de leucócitos, bem como por concentrações de proteína total e de fibrinogênio. Também foram verificados aumentos na concentração de lactato e de fósforo inorgânico no líquido peritoneal destes animais.

Utilizou-se um delineamento experimental inteiramente casualizado, com quatro grupos e dez coletas. Quando se constatou significância entre os grupos, dentro de cada momento, aplicou-se o teste de Tukey $(\mathrm{P}<0,05)$ para comparação das médias (SAMPAIO, 2002), por meio do programa estatístico SAS ${ }^{\circ}$.

\section{RESULTADOS E DISCUSSÃO}

Os resultados dos valores de $\mathrm{pH}(\mathrm{v}), \mathrm{pO}_{2}(\mathrm{v})$, $\mathrm{pCO}_{2}(\mathrm{v}), \mathrm{cHCO}_{3}^{-}(\mathrm{vP}), \mathrm{cBase}(\mathrm{v}), \mathrm{ctCO}_{2}(\mathrm{v}) \mathrm{e} \mathrm{sO}_{2}(\mathrm{v}) \mathrm{com}$ as respectivas médias, desvios-padrão e estatística calculada estão expressos nas tabelas 1 e 2 .
Nos primeiros 30 minutos de obstrução (T30ob), observou-se discreto aumento nos valores do $\mathrm{pH}(\mathrm{v})$ nos eqüinos do GIV, o qual, associado com o aumento da $\mathrm{CHCO}_{3}^{-}(\mathrm{vP})$ e da cBase(v) (Tabela 1), confirmou a alcalose metabólica. Esse aumento transitório do $\mathrm{pH}(\mathrm{v})$ foi ocasionado pela perda de íons potássio do sangue (hipocalemia). Quando os níveis de potássio do líquido extracelular estão baixos, o potássio move-se do líquido intracelular para o extracelular e é substituído em parte por íons hidrogênio que são perdidos do plasma. A perda de íons hidrogênio do organismo libera tampão, de modo que a concentração plasmática de $\mathrm{HCO}_{3}^{-}$e a base-tampão total aumentam, resultando na alcalose metabólica (ROBINSON, 2004).

A possível causa da hipocalemia apresentada pelos animais do GIV no T30ob foi a excitação, a dor e o estresse decorrentes da fase de indução das obstruções. Segundo FETTMAN (2004), esses fatores desencadeiam a liberação de hormônios como a insulina e as catecolaminas, as quais conseqüentemente induzem a hipocalemia.

Resultados semelhantes foram observados por SVENDSEN et al. (1979), DABAREINER \& WHITE (1995) e por RIBEIRO FILHO et al. (2007), entretanto, estes últimos autores incriminaram a administração da furosemida, uma das substâncias utilizadas no modelo de compactação do cólon maior, no desencadeamento da alcalose metabólica. Além disso, RIBEIRO FILHO et al. (2007) observaram que, com a evolução do quadro, esses mesmos animais passaram a apresentar acidose metabólica, que teve como provável origem a produção aumentada de lactato durante glicólise anaeróbica decorrente da hipovolemia, como foi descrito por HJORTKJAER \& SVENDSEN (1979) e.por NAPPERT \& JOHNSON(2001).

No T60ob, observou-se aumento dos valores do $\mathrm{pH}(\mathrm{v})$ nos eqüinos do GII, que ainda se mantiveram no T90ob e no T120ob, que teve como provável origem a perda de íons $\mathrm{H}^{+}$do conteúdo gástrico. A perda de íons $\mathrm{H}^{+}$também envolve a perda de íons cloretos, resultando na retenção do próximo íon negativo mais abundante no organismo, o $\mathrm{HCO}_{3}^{-}$. $\mathrm{O}$ resultado é um excesso de $\mathrm{HCO}_{3}^{-}$e o desencadeamento da alcalose metabólica (DAY, 2002). Corroborando as afirmações anteriores, observou-se aumento da $\mathrm{CHCO}_{3}^{-}(\mathrm{vP})$ e da cBase(v) nos animais do referido grupo e tempos, confirmando a alcalose metabólica (Tabelas 1 e 2). Neste ensaio, nos T150ob e T180ob, somente os animais do GII apresentaram refluxo gástrico por meio de sonda nasofaringeana. A quantidade de fluido obtido foi de aproximadamente um a 1,5 litros contendo principalmente partículas 
Tabela 1 - Média \pm desvio padrão do $\mathrm{pH}$ do sangue venoso - pH(v), pressão parcial do dióxido de carbono do sangue venoso - pCO ${ }_{2}(\mathrm{v})$, concentração total de dióxido de carbono no plasma do sangue venoso - $\mathrm{CtCO}_{2}(\mathrm{v})$ e concentração de bicarbonato no plasma do sangue venoso - $\mathrm{cHCO}$ $3(\mathrm{vP})$, dos animais dos grupos I ( $\mathrm{n}=6)$, II $(\mathrm{n}=6)$, III $(\mathrm{n}=6)$ e IV $(\mathrm{n}=6)$.

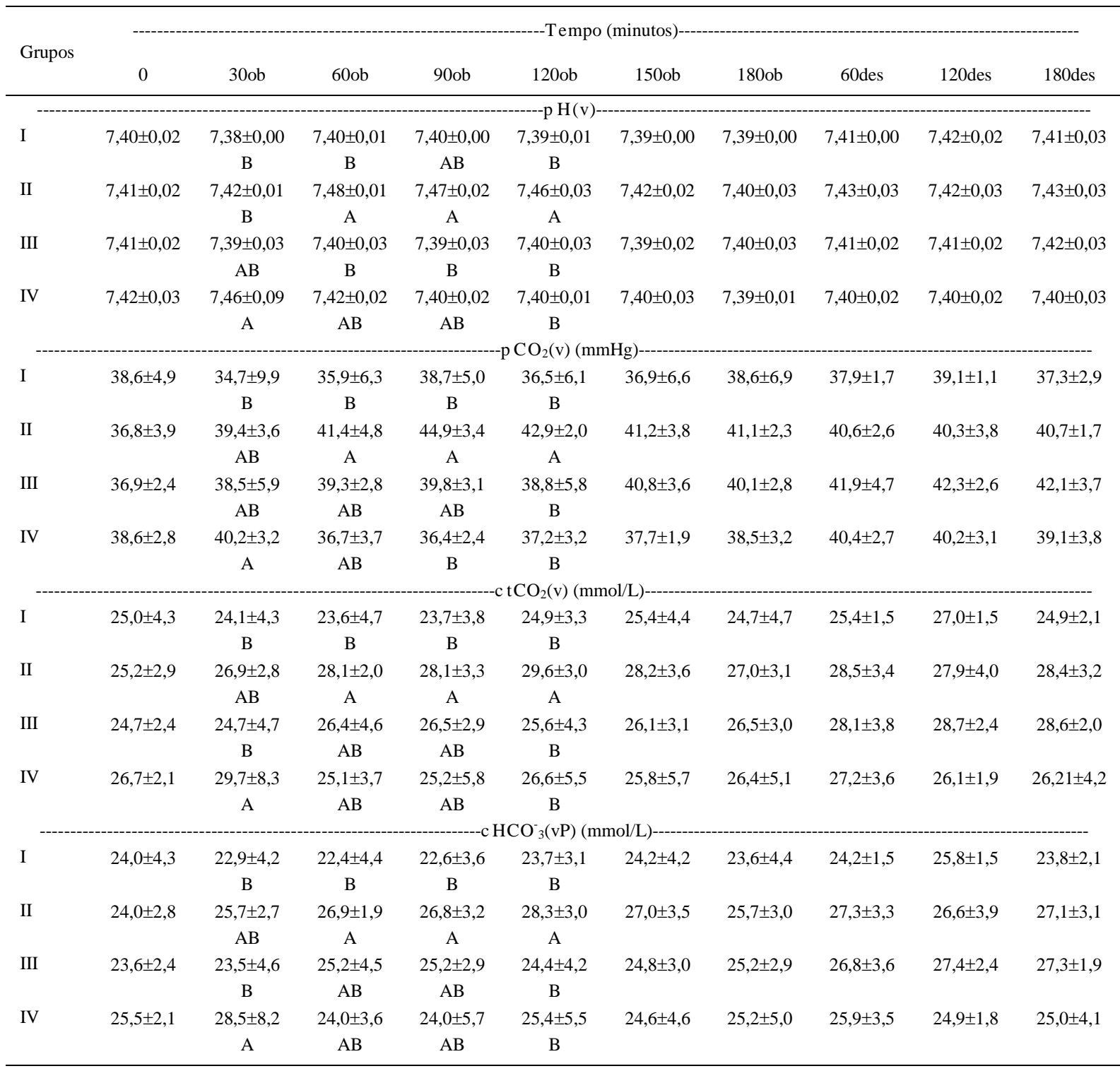

0: basal; 30ob-180ob: período de obstrução; 60des-T180des: período de desobstrução; GI=controle; GII=obstrução de duodeno; GIII=obstrução de íleo; GIV=obstrução de cólon maior. Médias seguidas por letras diferentes, na mesma coluna, diferem entre si pelo teste de Tukey, em nível de significância de 5\% e estabelecem comparação entre os diferentes grupos em cada momento.

alimentares, de coloração verde, odor fermentado, e com $\mathrm{pH}$ entre quatro e cinco. Diferindo dos resultados obtidos por PUOTUNEN-REINERT \& HUSKAMP (1986), a perda de fluidos por meio do refluxo gástrico não acarretou alterações no estado de hidratação dos animais, provavelmente em função do menor tempo de manutenção das obstruções, como foi descrito por NAPPERT \& JOHNSON (2001).

Em estudo realizado por PUOTUNENREINERT \& HUSKAMP (1986), três dos quatros animais submetidos à obstrução experimental do duodeno também apresentaram alcalose metabólica. Esses resultados assemelham-se aos obtidos por DATT \& USENIK (1975), os quais adicionalmente observaram que, em função da intensificação do desequilíbrio hídrico, esses mesmos animais passaram a apresentar acidose metabólica, mecanismo já explicado anteriormente.

Na tabela 1, observa-se no T30ob, nos animais do GIV, e nos T60ob, T90ob e T120ob, nos 
Tabela 2 - Média \pm desvio padrão da concentração de base titulável do sangue venoso - cBase(v), pressão parcial de oxigênio do sangue venoso $\mathrm{pO}_{2}$ (v) e saturação de oxigênio - $\mathrm{sO}_{2}(\mathrm{v})$ dos animais dos grupos I ( $\left.\mathrm{n}=6\right)$, II ( $\left.\mathrm{n}=6\right)$, III ( $\left.\mathrm{n}=6\right)$ e IV ( $\left.\mathrm{n}=6\right)$.

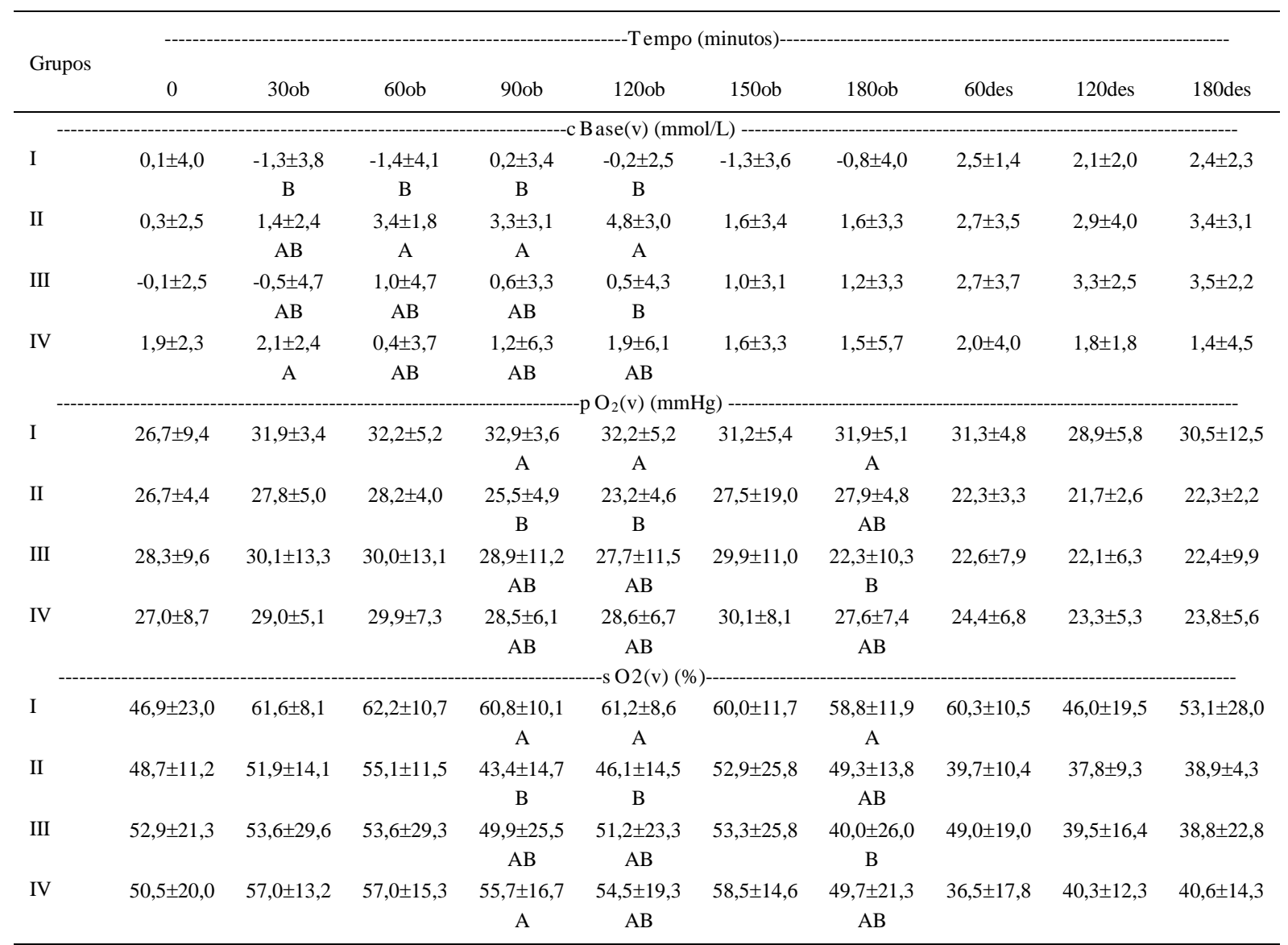

0: basal; 30ob-180ob: período de obstrução; 60des-T180des: período de desobstrução; GI=controle; GII=obstrução de duodeno; GIII=obstrução de íleo; GIV= obstrução de cólon maior. Médias seguidas por letras diferentes, na mesma coluna, diferem entre si pelo teste de Tukey, em nível de significância de $5 \%$ e estabelecem comparação entre os diferentes grupos em cada momento.

animais do GII, aumento da $\mathrm{pCO}_{2}(\mathrm{v})$, caracterizando a acidose respiratória. Segundo JOHNSON (1995), acidose respiratória geralmente ocorre como mecanismo de compensação imediata da alcalose metabólica ou como alteração primária nas afeç̧ões pulmonares. A acidose respiratória, desencadeada pela hipoventilação, faz com que o dióxido de carbono seja eliminado de maneira incompleta pelos pulmões, provocando um aumento na $\mathrm{pCO}_{2}$ sangüínea. Conseqüentemente, ocorre acúmulo de íons $\mathrm{H}^{+}$e, apesar do acúmulo simultâneo de bicarbonato, o pH diminui, pois a quantidade de bicarbonato acumulada é muito pequena para manter a proporção em um valor normal de 20:1 (DAY, 2002). Corroborando essas afirmações, havia alcalose metabólica confirmada pela $\mathrm{CHCO}_{3}^{-}(\mathrm{vP})$ e cBase(v) nos referidos grupos e tempos (Tabelas $1 \mathrm{e} 2$ ).
Os pulmões, juntamente com os rins, são os principais órgãos envolvidos na regulação do equilíbrio ácido-base. As compensações respiratórias ocorrem quase que imediatamente, pois, segundo ROBINSON (2004), os quimiorreceptores respondem rapidamente às alterações no $\mathrm{pH}$ sanguíneo, modificando rapidamente a p $\mathrm{CO}_{2}$. Entretanto, a resposta respiratória compensatória ocorre por curto período de tempo e permite a correção apenas de distúrbios leves. A correção a longo prazo requer a excreção de íons $\mathrm{H}^{+} \mathrm{e}$ a retenção de bicarbonato pelos rins (FETTMAN, 2004).

Corroborando as afirmações anteriores e considerando que o $\mathrm{pH}$ normal de referência do sangue venoso de eqüinos encontra-se entre 7,32 e 7,44 (CARLSON, 1997), constatou-se que as leves alterações apresentadas pelos animais ensaiados foram rapidamente corrigidas por modificações respiratórias. 
Diferindo dos resultados obtidos por NAPPERT \& JOHNSON (2001) e por DI FILIPPO et al. (2008), os quais verificaram que em função da gravidade do distúrbio abdominal os animais não foram capazes de corrigir as alterações, provavelmente devido ao comprometimento da integridade e perfusão renal, como foi descrito por DI FILIPPO \& SANTANA, (2007).

Nos animais do GII e do GIII, a $\mathrm{pO}_{2}(\mathrm{v})$ e a $\mathrm{SO}_{2}(\mathrm{v})$ tiveram comportamento semelhante (Tabela 2). Nos T90ob e T120ob, nos animais do GII, e no T180ob, nos animais do GIII, ocorreu diminuição nos seus valores. Esses valores baixos apresentados pelos animais do GII foram decorrentes do aumento da $\mathrm{pCO}_{2}$ ou da hipoventilação (Tabela 1), visando à correção da alcalose metabólica, mecanismo descrito anteriormente. Resultados semelhantes foram obtidos por RIBEIRO FILHO et al. (2007) e por DI FILIPPO et al. (2008). Entretanto, a diminuição da $\mathrm{pO}_{2}$ observada por RIBEIRO FILHO et al. (2007) foi associada à ocorrência de um processo inflamatório pulmonar, detectado em três dos animais ensaiados. A possível causa do processo inflamatório pulmonar foi o estresse presente nas fases de indução e no tratamento da compactação do cólon maior.

Nos animais do GIII, a hipoxemia observada foi oriunda da hipovolemia presente neste período. A manutenção e a intensificação do desequilíbrio hídrico, caso as obstruções intestinais fossem mantidas por mais tempo, daria origem à acidose metabólica, como foi verificado por DATT \& USENIK (1975) e por HJORTKJAER \& SVENDSEN (1979). Em ensaio realizado por DATT \& USENIK (1975), a acidose metabólica teve início 12 horas após a realização das obstruções e, com exceção dos animais com obstrução do cólon menor, todos os animais com obstrução de íleo e de duodeno foram a óbito.

$\mathrm{A} \mathrm{ctCO}_{2}(\mathrm{v})$ é a expressão da reserva alcalina, sendo o bicarbonato o seu principal componente. A diminuição na sua concentração é indicativa de acidose metabólica, enquanto o seu aumento é indicativo de alcalose metabólica (FETTMAN, 2004). Nos grupos II e IV, a ctCO (v) e a cBase(v) tiveram comportamento semelhante (Tabelas 1 e 2). No T30ob, nos animais do GIV e, nos T60ob, T90ob e T120ob, nos animais do GII, houve aumento nas suas concentrações, demonstrando alcalose metabólica.

Apesar do acúmulo de informações sobre a fisiopatologia das lesões de reperfusão no trato gastrintestinal de eqüinos (HORNE et al., 1994; MOORE et al., 1994; FALEIROS, 2001), não foram observadas alterações no equilíbrio ácido-base nos eqüinos dos grupos I, II, III e IV, nos T60des, T120des e T180des.

\section{CONCLUSÕES}

Com exceção dos eqüinos com obstrução de íleo, os animais com obstrução de duodeno e de cólon maior apresentam, na fase inicial do processo obstrutivo, alcalose metabólica. Tratam-se de alterações leves e temporárias, as quais por assim serem são rapidamente corrigidas por modificações respiratórias, não necessitando de qualquer intervenção terapêutica. As alterações metabólicas verificadas não são capazes de predizer o diagnóstico de obstruções intestinais específicas em eqüinos com cólica. Entretanto, auxiliam no prognóstico, visto que se relacionam diretamente com a precocidade do distúrbio gastrintestinal.

\section{AGRADECIMENTO}

À Fundação de Amparo à Pesquisa do Estado de São Paulo (Fapesp), pelo financiamento integral a esta pesquisa (processos no 05/58712-0 e 06/55377-8).

\section{COMITÊ DE ÉTICA E BIOSSEGURANÇA}

Aprovado pela Comissão de Ética e Bem-estar Animal (CEBEA), protocolo nํㅜ 023232-05.

\section{FONTES DE AQUISIÇÃO}

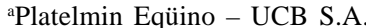

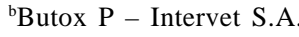

'Tec Horse - Purina.

${ }^{\mathrm{d} O m o l e n ~ E p h o s ~-~ P u r i n a . ~}$

${ }^{\mathrm{e} A c e p r a n}$ 1\% - Univet S.A.

${ }^{\text {f}}$ Virbaxil 2\% - Virbac.

sDolosal - Cristália.

hLidovet - Bravet.

iNeocaína 0,75\% - Cristália.

jTramal - Cristália.

kFlunixina Injetável - UCB S.A

'Pentabiótico Veterinário Reforçado - Fort Dodge.

mParinex - Hipolabor.

${ }^{\mathrm{n} A G S-12 ~-~ D r a k e . ~}$

oStatistical Analysis of System - versão 8.

\section{REFERÊNCIAS}

BRONDANI, J.T. et al. Alterações cardiovasculares de gatos submetidos à toracotomia intercostal pré-medicados com associação de tramadol, butorfanol e atropina e anetesiados com propofol e halotano. Ciência Rural, Santa Maria, v.33, n.5, p.869-873, 2003.

CARLSON, G.P. Fluid, electrolyte, and acid-base balance. In: KANEKO, J.J. et al. Clinical biochemistry of domestic animals. 4. ed. San Diego: Academic, 1997. Cap. 18, p. 485513.

DABAREINER, R.M.; WHITE, N.A. Large colon impaction in horses: 147 cases (1985-1991). Journal American 
Veterinary Medical Association, Schaumburg, v.206, n.5, p.679-685, 1995.

DATT, S.C.; USENIK, E.A. Intestinal obstruction in the horse. Physical signs and blood chemistry. Cornell Veterinarian, Ithaca, v.65, n.2, p.152-172, 1975.

DAY, T.K. Blood gas analysis. Veterinary Clinics of North America: Small Animal Practice, Philadelphia, v.32, n.5, p.1031-1048, 2002.

DI FILIPPO, P.A. et al. Equilíbrio ácido-base e hidroeletrolítico em eqüinos com cólica. Ciência Rural, Santa Maria, v.38, n.4, p.1003-1009, 2008.

DI FILIPPO, P.A; SANTANA, A.E. Variações nas concentrações dos biomarcadores sanguíneos da função renal e hepática em eqüinos com cólica. Veterinária Notícias, Goiânia, v.13, n.2, p.47-54, 2007.

FALEIROS, R.R. et al. Experimental ischemia and reperfusion in equine small colon. Arquivo Brasileiro de Medicina Veterinária e Zootecnia, Belo Horizonte, v.53, n.3, p.341350, 2001.

FETTMAN, M.J. Fluid and electrolyte metabolism. In: THRALL, M.A. et al. Veterinary hematology and clinical chemistry. Philadelphia: Lippincott Williams \& Wilkins, 2004. Cap.22, p. 329-355.

GOSSETT, K.A. et al. Contribution of whole blood L-lactate, pyruvate, D-lactate, acetoacetate, and 3-hydroxybutirate concentrations to the plasma anion gap in horses with intestinal disorders. American Journal of Veterinary Research, Schaumburg, v.48, n.1, p.72-75, 1987.

HJORTKJAER, R.K; SVENDSEN, C.K. Simulated small intestinal volvulus in the anesthetized horse. Nordisk Veterinaermedicin, København, v.31, n.11, p.466-483, 1979.

HORNE, M.M. et al. Attempts to modify reperfusion injury of equine jejunal mucosa using dimethylsulfoxide, allopurinol, and intraluminal oxygen. Veterinary Surgery, Philadelphia, v.23, n.4, p.241-249, 1994.

JOHNSON, P.J. Electrolyte and acid-base disturbances in the horse. Veterinary Clinics of North America: Equine practice, Philadelphia, v.11, n.3, p.491-514, 1995.
LARSEN, J. Acid-base and electrolyte balance in horses with various gastrointestinal disorders. In: EQUINE COLIC RESEARCH SYMPOSIUM, 5., 1994, Athens, GE. Proceedings... Athens: University of Georgia, 1994. p. 9.

MOORE, R.M. et al. Histopatological evidence of reperfusion injury in the large colon of the horse after low-flow ischemia. American Journal of Veterinary Research, Schaumburg, v.55, n.10, p.1434-1443, 1994.

NAPPERT, G.; JOHNSON, P.J. Determination of the acid-base status in 50 horses admitted with colic between December 1998 and May 1999. Canadian Veterinary Journal, Ottawa, v.42, n.9, p.703-707, 2001.

NATALINI, C.C.; ROBINSON, E.P. Evaluation of the analgesic effects of epidurally administered morphine, alfentanil, butorphanol, tramadol and $\mathrm{U} 50488 \mathrm{H}$ in horses. American Journal of Veterinary Research, Schaumburg, v.61, n.12, p.1579-1586, 2000.

PUOTUNEN-REINERT, A.; HUSKAMP, B. Experimental duodenal obstruction in the horse. Veterinary Surgery, Philadelphia, v.15, n.6, p. 420-428, 1986.

RIBEIRO FILHO, J.D. et al. Hemogasometria em eqüinos com compactação experimental do cólon maior tratados com sene, fluidoterapia enteral e parenteral. Ciência Rural, Santa Maria, v.37, n.3, p.755-761, 2007.

ROBINSON, N.E. Homeostase ácido-básica. In: CUNNINGHAM, J.G. Tratado de fisiologia veterinária. Rio de Janeiro: Guanabara Koogan, 2004. Cap.51, p.539-550.

SAMPAIO, I.B.M. Estatística aplicada à experimentação animal. 2.ed. Belo Horizonte: FEPMVZ, 2002. 265p.

SPEIRS, C.V. The alimentary tract. In: Clinical examination of horses. Philadelphia: Saunders, 1997. p.261298.

SVENDSEN, C.K. et al. Colic in the horse: a clinical and clinical chemical study of 42 cases. Nordisk Veterinaermedicin, København, v.31, n.10, p. 1-32, 1979.

TURNER, A.S.; McILWRAITH, C.W. Laparotomia do flanco e exploração abdominal. In: _____. Técnicas cirúrgicas em animais de grande porte. São Paulo: Roca, 2002. p. 237-242. 